\title{
Criminologie
}

\section{Les méthodes qualitatives en " terrain criminologique " : mise en perspective et usage de ces méthodes dans la revue Criminologie}

Qualitative methods in the criminological field: perspectives and use in Criminologie

\section{Los métodos cualitativos en "campo criminológico": Puesta en perspectiva y lugar de estos métodos en la revista Criminologie}

\author{
Jean Poupart et Amélie Couvrette
}

Volume 51, numéro 1, printemps 2018

50 ans de Criminologie

URI : https://id.erudit.org/iderudit/1045313ar

DOI : https://doi.org/10.7202/1045313ar

Aller au sommaire du numéro

Éditeur(s)

Les Presses de l’Université de Montréal

ISSN

0316-0041 (imprimé)

1492-1367 (numérique)

Découvrir la revue

Citer cet article

Poupart, J. \& Couvrette, A. (2018). Les méthodes qualitatives en « terrain criminologique " : mise en perspective et usage de ces méthodes dans la revue Criminologie. Criminologie, 51(1), 201-229. https://doi.org/10.7202/1045313ar

\section{Résumé de l'article}

L'objectif de cet article est de décrire la place et l'usage des méthodes qualitatives tels qu'ils ressortent des recherches qui ont fait l'objet d'articles dans la revue Criminologie. Pour ce faire, nous nous intéressons au développement de ces méthodes à l'École de criminologie de l'Université de Montréal, puis à certaines des transformations qui ont façonné le champ de la criminologie et celui de la recherche qualitative dans leur ensemble et, enfin, aux pratiques de recherches qualitatives diffusées dans la revue. Le corpus retenu comprend 114 articles qualitatifs publiés entre 1972 et 2016. L’analyse permet de dresser un portrait des usages de la méthodologie qualitative, d'exemplifier la façon dont sont rapportés ces usages et de cerner des tendances dont certaines sont communes à celles observées à l'École de criminologie, mais également, en criminologie. 


\title{
Les méthodes qualitatives en «terrain criminologique $»$ : mise en perspective et usage de ces méthodes dans la revue Criminologie
}

\author{
Jean Poupart \\ Professeur honoraire \\ École de criminologie et Programme de doctorat en sciences humaines appliquées \\ Université de Montréal \\ jean.poupart@umontreal.ca \\ Amélie Couvrette ${ }^{12}$ \\ Professeure \\ Département de psychoéducation et de psychologie, Université du Québec en Outaouais \\ Chercheure régulière, Centre international de criminologie comparée (CICC) \\ Chercheure associée, Centre de recherche de l'Institut universitaire \\ en santé mentale de Montréal (CRIUSMM) \\ amelie.couvrette@uqo.ca
}

RÉSUMÉ - L'objectif de cet article est de décrire la place et l'usage des méthodes qualitatives tels qu'ils ressortent des recherches qui ont fait l'objet d'articles dans la revue Criminologie. Pour ce faire, nous nous intéressons au développement de ces méthodes à l'École de criminologie de l'Université de Montréal, puis à certaines des transformations qui ont façonné le champ de la criminologie et celui de la recherche qualitative dans leur ensemble et, enfin, aux pratiques de recherches qualitatives diffusées dans la revue. Le corpus retenu comprend 114 articles qualitatifs publiés entre 1972 et 2016. L'analyse permet de dresser un portrait des usages de la méthodologie qualitative, d'exemplifier la façon dont sont rapportés ces usages et de cerner des

1. Département de psychoéducation et de psychologie, Université du Québec en Outaouais, 283, boulevard Alexandre-Taché, pièce C-2911, C. P. 1250, succ. Hull, Gatineau (Québec), Canada, J8X 3X7.

2. Nous sommes redevables à Michèle Lalonde, les premières sections de cet article s'inspirant de ses travaux antérieurs (Poupart et Lalonde, 1997). Nous la remercions également pour ses conseils à différents moments de notre recherche. Nous souhaitons également souligner le soutien de France Nadeau, de la BLSH de l'Université de Montréal, et le travail des étudiantes de l'UQO, Frédérique Hervieux et Olivia Levesque Côté.

Criminologie, vol. 51, $\mathrm{n}^{\circ} 1(2018)$ 
tendances dont certaines sont communes à celles observées à l'École de criminologie, mais également, en criminologie.

MotS CLÉs - Méthodes qualitatives et revue Criminologie, institutionnalisation des méthodes qualitatives en criminologie, débats épistémologiques, approches qualitatives.

\section{Introduction}

Dès sa création en 1968, la revue Criminologie s'est voulue un espace de diffusion des recherches menées à l'École de criminologie de l'Université de Montréal et au sein de la discipline. Que peut-on dire des recherches qui ont eu recours à une méthodologie qualitative et qui ont été publiées dans la revue? Nous examinerons cette question en mobilisant trois éclairages: 1) celui de l'émergence et du développement, dès les années 1970, des méthodes qualitatives à l'École de criminologie; 2) celui de quelques-unes des transformations qui sont survenues au cours des années 1990 et 2000, non seulement au sein de la discipline, mais également dans le domaine des méthodes qualitatives; 3) celui des recherches qualitatives diffusées dans la revue Criminologie sous l'angle de leurs aspects méthodologiques.

Ces divers éclairages permettront de mieux cerner l'usage des méthodes qualitatives en ce qui a trait à leurs fondements théoriques et épistémologiques, aux diverses approches utilisées et à leur(s) justification(s), aux différents angles par lesquels les objets sont appréhendés, de même qu'en ce qui concerne les différentes composantes des démarches de recherche, allant de la description du type d'entretien ou d'observation aux questions de validité et de généralisation.

D'entrée de jeu, une remarque s'impose sur ce qu'il faut entendre par méthodes qualitatives. La réponse à cette question n'est pas aussi simple qu'elle n'y paraît puisque cette dénomination présente des frontières relativement floues mettant en jeu une variété de techniques, de pratiques, voire de conceptions de la recherche (Groulx, 1998). Une représentation fréquente est sans doute celle référant à un ensemble de modes de recueil et d'analyse de données tels ceux associés aux entretiens, à l'observation participante et à l'analyse documentaire, mais aussi à un éventail de démarches comme l'ethnographie, la théorisation ancrée, l'approche biographique et les études de cas. Pour plusieurs, les techniques et les différentes approches associées aux méthodes qualitatives sont liées à des postures théoriques et épistémologiques dont il est impossible de faire abstraction, telle la place privilégiée à accorder aux perspectives des acteurs sociaux, point de vue que nous partageons. 


\section{1. Émergence et développement des méthodes qualitatives à l'École de criminologie comme formule de recherche}

L'usage des méthodes qualitatives dans les recherches publiées dans la revue Criminologie ne pouvant être compris isolément, nous reviendrons brièvement sur l'émergence et le développement à l'École de criminologie, au cours des années 1970 et 1980, de ces méthodes comme formule de recherche ${ }^{3}$, notion proposée par Chapoulie (1984) pour rendre compte de l'élaboration et de la mise en œuvre, comme démarche empirique, de l'observation participante au sein de la tradition de Chicago.

1.1 Les années 1960: une nouvelle discipline axée sur la mesure et la quantification

À l'instar des autres sciences sociales, dès sa création au début des années 1960, l'École s'est inscrite, en termes d'orientations, dans le courant positiviste. Elle se voudra axée sur l'étude des causes de la criminalité et de la délinquance et sur la prédiction et l'évaluation des mesures en place (Ellenberger, 1965; Szabo, 1963). La recherche, à visée pragmatique, devra servir à fonder les pratiques d'intervention et les politiques pénales.

Les travaux entrepris alors à l'École seront essentiellement axés sur la mesure et la quantification. Le statut de la jeune discipline étant mal assuré, c'est par la méthodologie qu'Ellenberger (1965) et Szabo (1963) entendront en garantir la scientificité par l'utilisation des «instruments de mesure les plus modernes» (Szabo, 1963). Dans ce contexte, il n'y aura guère de place pour les méthodes qualitatives, celles-ci ne faisant pas partie des instruments dits modernes.

\subsection{Les années 1970 et 1980: implantation des méthodes qualitatives}

Pratiquement ignorées dans les années 1960, les méthodes qualitatives s'implanteront rapidement durant les années 1970 et 1980, comme en témoignent les changements dans les discours, la multiplication des

3. Pour une analyse plus détaillée, voir Poupart et Lalonde (1997) et, à titre complémentaire, Poupart $(2004,2008,2011)$. 
recherches, l'instauration d'enseignements spécialisés et la publication d'articles sur ces méthodes.

Ainsi, sur le plan des discours, au tournant des années 1970, des professeurs de l'École feront valoir l'intérêt de la démarche ethnographique pour étudier certaines formes de déviance ou de criminalité. Par exemple, Normandeau et Szabo (1970) préconiseront le recours à une approche méthodologique pluraliste faisant une place à l'observation participante. Gagné (1970), quant à lui, soutiendra que si les études quantitatives permettent de montrer que la délinquance juvénile est présente ailleurs que dans les milieux défavorisés, l'observation participante serait davantage appropriée pour appréhender la sous-culture des jeunes «déviants». La recherche qu'il entreprendra sur la sousculture de jeunes Montréalais de classe moyenne impliqués dans la consommation et la vente de drogues, poursuivie par Le Blanc (1972), sera la première recherche qualitative publiée dans la revue. Szabo (1972) soulignera en éditorial le rôle essentiel de l'observation participante en criminologie:

L'observation participante seule permet la pénétration des chercheurs dans ces milieux et l'étude de Le Blanc illustre les multiples ressources de cette technique dans l'analyse sous-culturelle. Le recours plus systématique à cette technique, afin de décrire le fonctionnement d'un système social à caractère déviant et antisocial, apportera une lumière qui a manqué jusqu'à présent à cette notion complexe de la déviance. Ces travaux influenceront, à l'avenir, d'une manière décisive notre conception de la criminalité et du criminel en substituant aux jugements et aux évaluations binaires des analyses beaucoup plus nuancées. (p. 6)

Il ressort ici l'argument selon lequel seule une connaissance de l'intérieur permettrait d'appréhender les modes de vie, les façons d'agir et de penser de certains groupes. D'autres arguments seront mis de l'avant dans les travaux de Brillon (1980) sur le règlement des litiges en Côte d'Ivoire et de Finkler $(1975,1980)$ sur l'administration de la justice en milieu inuit : l'observation participante favoriserait, grâce à une immersion prolongée, une connaissance en profondeur des phénomènes et leur compréhension dans leur contexte social et culturel.

À leurs débuts, les méthodes ethnographiques seront souvent présentées de manière défensive, en faisant valoir «ce qu'elles permettent de faire ou de mieux faire» par rapport aux approches quantitatives. Ce mode de justification sera repris par les chercheurs qui, dans la seconde moitié des années 1970, auront recours aux entretiens de 
recherche. Plus fondamentalement, les approches qualitatives seront promues comme démarche de plein droit plutôt que comme dispositif d'appoint (voir l'article de Louis-Guérin [1979] publié dans la revue). Largumentation ne sera pas que défensive et prendra, dans plusieurs cas, la forme d'une critique virulente. Ainsi, dans leur étude sur les services novateurs réalisée par observation participante, Bertrand et al. (1972) refuseront de «tenir un discours sur ...», «de parler à la place de...» (p. 11), dénonçant «le placage réifiant d'un cadre théorique conçu de l'extérieur» (p. 42) et la prétendue neutralité du chercheur. Pour sa part, Tardif (1974) déclarera que les méthodes qualitatives sont celles à privilégier :

L'inventaire de qui a été écrit sur la police ainsi que mes propres recherches m'ont enseigné que dans le seul domaine qui pourrait prêter à une certaine quantification [...] les résultats sont tels qu'il nous faut continuer de retenir l'hypothèse nulle. Déjà, ceci devrait être de nature à choisir une autre méthode. Mais qu'en plus, l'on se propose d'étudier les rapports beaucoup plus difficilement chiffrables unissant la police à la politique ou ces deux éléments à la déviance et que, par surcroît, l'on se situe d'emblée dans une hypothèse interactionniste, ceci appelle une tout autre méthodologie que celle dérivée des sciences exactes. C'est à l'approche qualitative qu'il faut recourir, et non pas comme si c'était une méthode parmi plusieurs, un pis-aller juste à servir de phase exploratoire, d'étude-pilote, mais parce que c'est la méthode à utiliser dans les sciences de l'homme. (p. 21)

Si l'observation participante occupe au départ l'avant-scène, le recours aux entretiens de recherche deviendra prépondérant et les justifications avancées pour en faire usage seront similaires à celles de l'observation participante. Les études réalisées concerneront des objets diversifiés, incluant les services de police (Tardif, 1974), les libérations conditionnelles (Carrière, 1976; Nicolas, 1974), la détention des mineurs (Laflamme-Cusson et Baril, 1975), la criminalité des femmes (Bertrand, 1979), les représentations de la criminalité et du système de justice (Louis-Guérin, 1979), les victimes de vols à main armée (Huot et Giroux, 1979; Manseau et Grenier, 1979) ou de violence conjugale (Baril, Cousineau et Gravel, 1983), les coûts sociaux du système pénal (Blankevoort, Landreville et Pires, 1981), la dangerosité et la pratique criminologique (Poupart, Dozois et Lalonde, 1982). À ces travaux s'ajouteront des recherches menées à partir de sources documentaires sur la sociohistoire des institutions de régulation sociale (Laberge, 1983; Théorêt, 1987) ou sur le fonctionnement d'instances telles les commissions d'enquête (Acosta, 1986; Brodeur, 1984). 
En parallèle, émerge au sein de la criminologie québécoise une littérature spécialisée sur l'histoire des méthodes qualitatives, leurs fondements, leurs usages et les débats les entourant. Ainsi, à l'instar de ce qui se passe dans d'autres disciplines au Québec, ces méthodes deviendront en elles-mêmes un champ d'études.

\subsection{Mise en place d'enseignements spécialisés}

Le développement à l'École de recherches effectuées à partir d'une méthodologie qualitative sera soutenu par la mise en place dès le début des années 1970 d'enseignements spécialisés. Signe de l'acceptation de cette méthodologie par le corps professoral, ces enseignements, qui en légitiment le recours, joueront un rôle essentiel comme lieu de transmission des savoirs théoriques et pratiques. Ils seront l'occasion pour de nombreux étudiants d'entreprendre leurs travaux de recherche.

Deux sources d'influence façonneront ces enseignements et la manière de mettre en œuvre une approche qualitative. La première est reliée à la sociologie américaine et, en particulier, à la tradition de Chicago. Elle se fera sentir non seulement sur le plan des fondements, mais également sur celui de l'apprentissage des méthodes qualitatives, en mettant l'accent sur l'importance de s'y initier «en faisant du terrain».

La seconde source d'influence découle des travaux sur les représentations sociales de la justice menés en France par Robert et Faugeron (1978) à partir d'entretiens à tendance non directive, travaux largement diffusés dans la criminologie francophone. En 1977, Faugeron donnera à l'École une formation sur les principes, les stratégies et les techniques à mettre en œuvre dans la conduite et l'analyse de ce type d'entretien. Cette formation, à base d'exercices pratiques, sera marquante dans l'enseignement des méthodes de recherche qualitatives à l'École et ailleurs dans la francophonie (Kaminski et Kokoreff, 2004 ; Pierret, 2004; Pires, 2004). Bien que plusieurs considérations puissent intervenir dans le choix des différentes méthodes en méthodologie qualitative comme celles relatives à l'objet de l'étude, aux exigences propres à chaque méthode et à leur statut dans la communauté scientifique, ce type d'enseignement n'est sans doute pas étranger à la popularité que connaîtront les entretiens comme dispositif de recherche en criminologie. 


\subsection{Débats autour des méthodes: une question de paradigmes?}

Au milieu des années 1980, les méthodes qualitatives se sont institutionnalisées à l'École et font désormais partie de la «science normale». D'usage courant, elles sont devenues une formule de recherche reconnue, c'est-à-dire un mode de connaissance empirique légitime, valorisé et crédible scientifiquement. Loin d'être unique, une telle situation s'observe dans d'autres milieux de la criminologie comme dans l'ensemble des sciences sociales, au Québec et sur le plan international. Ce «retour» des méthodes qualitatives comme dispositif de recherche est à mettre en relation avec la critique particulièrement forte dans les années $1970 \mathrm{du}$ modèle positiviste et des approches quantitatives. De plus, ce dispositif deviendra acceptable du point de vue des critères habituels de la scientificité, à la suite de travaux comme ceux de Glaser et Strauss (1967), montrant qu'elles peuvent être utilisées de manière rigoureuse et systématique sur les plans du recueil et de l'analyse des données, de la construction de l'échantillon et des formes de généralisation.

À l'École de criminologie et, plus largement, au sein de la discipline, la montée des méthodes qualitatives est également liée à l'influence de la sociologie de la déviance interactionniste et ethnométhodologique, à l'origine des thèses de la réaction et du contrôle social. Des clivages sur les plans idéologique, épistémologique et théorique en découleront. S’opposeront une criminologie positiviste axée sur l'étude étiologique de la délinquance et une criminologie de la réaction sociale centrée sur l'étude des processus de définition du crime, des mécanismes de régulation sociale et leur impact quant à l'identité et à la trajectoire des déviants.

Pendant plus de 25 ans, les débats au sein de la discipline s'articuleront autour de ces conflits de paradigmes, auxquels se grefferont les questions de méthodologie. Ainsi, dans la perspective de la réaction sociale, les méthodes qualitatives apparaîtront comme une façon nouvelle, différente, de faire de la criminologie. À cet égard, une plus grande place sera accordée aux perspectives et aux expériences des acteurs «déviants», considérés comme des informateurs clés sur le fonctionnement des institutions de contrôle social. Ensuite, les approches qualitatives seront abondamment utilisées pour investiguer, sous l'angle de la réaction sociale, des objets tels que les enjeux normatifs autour de la déviance, les représentations de la justice, les idéologies et les pratiques professionnelles et l'analyse des diverses instances du système pénal.

Les oppositions entre méthodes qualitatives et quantitatives à l'École s'estomperont à partir de la fin des années 1980. En dépit de ces oppo- 
sitions, les méthodes qualitatives seront très tôt employées selon une diversité d'approches et d'orientations. L'accent sera également mis sur la complémentarité des méthodes, celles-ci étant vues comme permettant d'étudier différentes choses et les mêmes choses différemment. En outre, le débat qualitatif et quantitatif sera rapidement considéré comme un faux débat, les divergences en sciences sociales étant vues comme tenant moins au choix des méthodes qu'aux orientations théoriques des chercheurs et à leurs conceptions de la science.

\section{2. ( $R e$ ) positionnement au sein de la discipline et des méthodes qualitatives}

Les controverses quant à l'objet de la criminologie s'atténueront au cours des années 1990. Envisager la question criminelle selon une approche en termes de réaction sociale ne suscitera plus autant de réticences. De plus, au sein même de cette approche, on assiste à un repositionnement par rapport à l'étude de l'étiologie de la délinquance. Pires (1993, 1995), dont les travaux sont exemplaires de ce repositionnement, soutiendra qu'il est possible d'étudier les processus d'implication dans les conduites socialement réprouvées sans adopter une vision substantive de ces conduites et en prenant en compte les normativités qui les entourent. De plus, pour Pires (1998), les perspectives épistémologiques sur lesquelles se fondent les recherches devraient dépendre de la nature des objets à l'étude, les divergences entre postures positiviste et constructiviste étant en outre moins tranchées qu'elles ne le paraissent, voire comme menant à de fausses oppositions.

Ce repositionnement sur le plan des paradigmes s'accompagne de changements quant aux problématiques en criminologie. Ainsi, avec la montée du néolibéralisme et du conservatisme dans les années 1980 et 1990, une question désormais débattue dans la discipline sera celle de savoir s'il y a lieu, en termes de politiques pénales, de maintenir les acquis de l'État social ou plutôt d'adopter des mesures plus répressives envers les contrevenants (Garland, 1981; Wincup, 2017). De plus, comme le reflète la revue, certaines thématiques liées à la conjoncture des années 2000 deviendront plus prégnantes, comme le génocide, les crimes de guerre, le terrorisme, l'immigration illégale et les questions d'ordre et de sécurité ${ }^{4}$.

4. Ces thématiques sont aussi à mettre en rapport avec la mise en place du programme de sécurité et d'études policières à l'École de criminologie. 
2.1 Déplacement des controverses et des interrogations dans le champ des méthodes qualitatives

À partir des années 1990, on assiste à un déplacement des controverses dans le champ même des méthodes qualitatives. Ce déplacement, visible dans la littérature anglo-saxonne, est illustré par The SAGE handbook of qualitative research, publié en 1994 sous la direction de Denzin et Lincoln et réédité à plusieurs reprises. Sous l'influence du postmodernisme et du constructivisme, Denzin et Lincoln rejettent radicalement le modèle positiviste et celui, plus nuancé, du postpositivisme selon lequel il est possible de parvenir à une connaissance approchée de la réalité. Pour ces auteurs, le réel ne pouvant être saisi que par de multiples perspectives, le rôle de la science devient celui de déconstruire et de reconstruire les différentes lectures en présence, de les confronter et de les mettre en dialogue pour éclairer l'action. De ce point de vue, des travaux comme ceux de Glaser et Strauss (1967) et de Miles et Huberman (1994), fortement associés au développement des méthodes qualitatives, seront considérés comme d'orientation trop positiviste.

Ces réflexions d'ordre épistémologique s'accompagneront d'interrogations de nature méthodologique concernant les conceptions et les processus de recherche en sciences sociales. Parmi celles-ci, mentionnons la place de la subjectivité dans la production et l'interprétation des données et l'influence qu'y exercent les caractéristiques de genre, de position sociale, d'appartenance culturelle ou ethnique; l'importance à conférer à la parole des acteurs de différents courants comme le «standpoint» associé aux perspectives féministes, les «multivoice studies», la recherche-action et les «culturals studies»; les critères pour juger de la validité de la recherche; la pertinence de l'engagement des chercheurs ou les dimensions éthique et sociopolitique des études. Ces interrogations se reflètent par exemple dans le collectif de Lumsden et Winter (2014) traitant des méthodes qualitatives en criminologie, les auteurs invitant les chercheurs à faire preuve de réflexivité par rapport à ces interrogations.

\subsection{L'ethnographie: compte rendu de recherche, récit ou narration?}

Ces dernières interrogations portent également sur le statut des récits ethnographiques. Jusqu'à quel point les chercheurs peuvent-ils rendre compte des perspectives et des expériences des acteurs sociaux, les 
méthodes qualitatives ayant toujours été envisagées comme la voie royale pour y parvenir, comme dans la tradition de Chicago? La possibilité d'y arriver a fait l'objet d'incessantes préoccupations (Poupart, 1993, 1997). Dans les années 1950, on pensait pouvoir le faire par des précautions d'ordre strictement méthodologique visant à neutraliser les biais associés aux entretiens aussi bien qualitatifs que standardisés. À partir des années 1970, ces précautions apparaîtront insuffisantes, les discours étant vus comme socialement construits dans des contextes d'énonciation et des conditions inhérentes aux dispositifs de recherche, aux relations entre chercheurs et sujets interrogés et aux enjeux de la recherche. Dans cette perspective, les interviewés reconstruiraient leur réalité et les chercheurs feraient des reconstructions de ces reconstructions.

Les réflexions sur le statut des «récits» ethnographiques des chercheurs vont encore plus loin. Ces récits seront considérés par certains comme des «narrations». Une plus grande attention sera apportée à la manière dont les chercheurs se projettent, avec leurs présupposés, dans la production des récits et aux stratégies d'argumentation utilisées pour persuader les lecteurs de la véracité de leurs analyses. À la limite, pour certains comme Clough (1992), les interviewés raconteraient des histoires sur leur vie et les chercheurs raconteraient des histoires sur ces histoires. Vues sous cet angle, les narrations n'auraient guère de rapport avec la réalité.

Cette mise en question, minoritaire, des fondements des récits ethnographiques suscitera diverses réactions. Comme le rapportent Erickson (2018) et Denzin et Lincoln (2018), cette vision sera envisagée par des chercheurs comme niant les fondements mêmes des méthodes qualitatives. On trouve des traces de ces controverses dans la littérature francophone, notamment dans les travaux de Cefaï (2003) et d'Olivier de Sardan (2008), lequel rappelle que bien qu'on ne puisse échapper aux processus d'interprétation inhérents à toute recherche, il demeure fondamental de ne pas perdre de vue le «référent empirique» comme assise pour traduire et interpréter la réalité des acteurs sociaux. Dans la revue Criminologie, les auteurs adoptent une «posture réaliste», les articles visant à décrire ce qui se passe ou s'est passé, ce qui ne signifie pas que les chercheurs ignorent la part d'interprétation et de reconstruction que supposent ces «récits», ne serait-ce qu'en raison de l'influence du courant constructiviste en criminologie. 
Paradoxalement, au moment où les méthodes qualitatives sont l'occasion de réflexions sur les fondements de la connaissance scientifique, les conditions actuelles dans lesquelles se pratiquerait la recherche favoriseraient un retour en force du positivisme, contexte jugé moins favorable aux méthodes qualitatives. Selon Wincup (2017), Lumsden et Winter (2014), Erickson (2018) et Denzin et Lincoln (2018), si le point de vue dominant dans les milieux de la recherche aux États-Unis et en Grande-Bretagne restait celui de la complémentarité des méthodes et si les recherches mixtes étaient préconisées, les exigences gouvernementales et celles des organismes subventionnaires, dans le contexte sociopolitique actuel, favoriseraient un retour à une forme de positivisme «dur», par exemple dans l'usage jugé parfois trop restrictif des données probantes (evidence-based data). Seraient ainsi privilégiées les recherches, $\mathrm{y}$ inclus qualitatives, répondant aux exigences de ces institutions et à une prétendue rigueur scientifique, avec une tendance à favoriser les méthodes quantitatives.

Jusqu'à quel point cette reprise du débat, si souvent dénoncé, entre les méthodes qualitatives et quantitatives traverse-t-elle les milieux de la criminologie francophone? Cette question demeure à être examinée. Si tel n'est pas le cas, il n'y aurait pas lieu de s'en plaindre. Outre le fait qu'il ne faut pas ignorer ce débat, ne serait-ce que pour mieux s'en prémunir, il n'en est pas moins nécessaire que les chercheurs en criminologie, ici comme ailleurs, fassent preuve de réflexivité sur la manière dont les conditions sociales et institutionnelles dans lesquelles sont produites les recherches viennent marquer aussi bien le choix des objets que la façon dont ils sont traités. De plus, les débats sur les fondements de la connaissance et sur ce qui les affecte sont nécessaires, à moins de renoncer à s'interroger sur ce qu'implique faire de la recherche en criminologie et en sciences sociales, y compris sous l'angle des méthodes utilisées.

\section{Les études qualitatives publiées dans la revue Criminologie : caractéristiques et modes de présentation}

Cette section explore l'usage des méthodes qualitatives au sein de la revue Criminologie et vise à poursuivre l'analyse sur les usages des méthodes qualitatives, mais cette fois, en utilisant un corpus émanant spécifiquement de la revue. 


\subsection{Méthodologie}

Le corpus de l'étude comprend 114 articles empiriques, publiés dans la revue entre 1972 et 2016, ayant eu recours à la méthodologie qualitative. Ces articles ont été repérés sur la plateforme Érudit et importés dans le logiciel de gestion de références bibliographiques EndNote. Après avoir lu et catégorisé les articles selon le type de méthodologie utilisé, ceux-ci ont ensuite été transférés dans le logiciel d'analyse qualitative NVivo. Les principales sections des articles ont par la suite été lues et codifiées. En raison de l'ampleur du corpus, nous avons centré notre analyse principalement sur les sections méthodologiques, en recourant parfois, lorsque les sections méthodologiques présentaient certaines lacunes, aux résumés, à la description des objets d'étude en introduction et aux passages jugés pertinents de la conclusion. Il faut toutefois reconnaître que ce choix comporte certaines limites, dont de ne considérer que les informations traitées dans les sections analysées et d'écarter le contexte d'ensemble de l'article. Des questions avaient préalablement été formulées quant à l'usage de la méthode qualitative à partir de notre connaissance du champ, mais également de certains travaux ayant traité d'une problématique similaire (Baribeau et Royer, 2012; Royer, Baribeau et Duchesne, 2009). À l'aide de ces questions, nous avons décortiqué les éléments méthodologiques des articles consultés. Le logiciel NVivo a ensuite permis d'organiser et de regrouper les divers éléments de ces sections des articles composant notre corpus. Ces regroupements ont permis de saisir les récurrences et d'objectiver les éléments les plus souvent constitutifs des articles consultés. L'analyse subséquente a permis de réaliser une description détaillée des éléments méthodologiques de ces articles et de saisir comment les différents dispositifs de la méthodologie qualitative ont été utilisés. Trois autres éléments se sont également imposés lors de l'analyse: les justifications de la méthode, les approches théoriques méthodologiques choisies ainsi que l'aspect exploratoire de la recherche. Celles-ci seront abordées après avoir dressé un portrait de l'usage des méthodes qualitatives dans la revue.

Enfin, il importe de noter que le fruit de ces prochaines analyses permettra d'informer sur les diverses utilisations de la recherche qualitative dans cette revue spécialisée, mais qu'il ne représentera qu'un indice de leur développement, utilisation et légitimation des méthodes qualitatives dans le champ de la criminologie francophone. 


\subsection{Portrait général des articles}

Des 811 articles publiés dans la revue Criminologie depuis ses débuts, les articles qualitatifs représentent $14 \%$ de ses publications, soit 114 articles $^{5}$. Ces articles sont de deux ordres: des comptes rendus de recherches qualitatives sur un objet donné et des articles faisant référence à plusieurs études qualitatives, menées par les auteurs de l'article, dans le but d'illustrer ou de documenter certains aspects de l'objet de leur article. Le premier type d'articles compose la majorité du corpus bien que le second type représente une partie non négligeable des articles retenus.

D'emblée, un grand constat s'impose, et ce, peu importe le type d'article qualitatif. Il existe une grande disparité dans la façon dont les auteurs décrivent leur méthodologie. Certains auteurs ne fournissent que quelques détails en lien avec celle-ci, parfois en note en bas de page, alors que d'autres y consacrent quelques pages. Cette disparité complique l'analyse des méthodologies puisque ne sont pas rapportés les mêmes éléments méthodologiques d'un article à l'autre. Néanmoins, en plus d'avoir suscité une réflexion, la présence de cette disparité a permis d'enrichir notre analyse en présentant différents types de sections méthodologiques.

Le Tableau 1 présente les dispositifs de collecte de données privilégiés dans le corpus, et ce, depuis les premières publications de la revue en 1972. Il est possible de constater que le nombre d'articles qualitatifs publiés a augmenté pour atteindre son apogée lors de la dernière décennie. De plus, les articles utilisant l'entretien dans leur devis de recherche, comme dispositif principal ou complémentaire, représentent la majorité, soit $68 \%$, des articles. Un engouement certain pour ce dispositif est perceptible à travers les décennies. L'analyse documentaire et l'observation s'inscrivent comme dispositif privilégié dans respectivement $25 \%$ et $21 \%$ des études (comme technique principale ou complémentaire). Il est intéressant de noter que l'observation, à titre de dispositif principal, n'a été que peu utilisée dans le cadre de ces études. Ces constats se maintiennent par ailleurs dans le temps. Il importe de souligner l'emploi fréquent de méthodes combinées dans les articles consultés, plusieurs auteurs misant ainsi sur la complémentarité de ces

5. Outre les articles de méthodologie quantitative, lesquels représentent $23 \%$ de l'ensemble des publications (185 articles) (Charette, Tremblay et Deslauriers-Varin, 2018), les autres publications de la revue sont de l'ordre de l'éditorial, de commentaires critiques ou d'articles ne présentant pas de données empiriques. 
TABLEA U 1

Devis de recherche qualitatif et mixte des études consultées

en fonction de la décennie de publication

\begin{tabular}{|c|c|c|c|c|c|c|c|c|c|}
\hline & \multirow[b]{3}{*}{ Devis mixte ${ }^{1}$} & \multicolumn{7}{|c|}{ Devis qualitatif } & \multirow[b]{3}{*}{ Nombre total } \\
\hline & & \multicolumn{3}{|c|}{ Technique privilégiée } & \multicolumn{4}{|c|}{ Multiméthodes } & \\
\hline & & Entretien & Observation & Analyse doc & Entretien + obs & $\begin{array}{l}\text { Entretien + } \\
\text { analyse doc }\end{array}$ & $\begin{array}{c}\text { Obs + } \\
\text { analyse doc }\end{array}$ & $\begin{array}{l}\text { Entretien + obs } \\
+ \text { analyse doc }\end{array}$ & \\
\hline $1970-1979$ & 0 & 3 & 1 & 0 & 1 & 0 & 0 & 0 & 5 \\
\hline $1980-1989$ & 1 & 7 & 0 & 2 & 0 & 0 & 0 & 2 & 12 \\
\hline 1990-1999 & 3 & 2 & 0 & 2 & 0 & 1 & 0 & 2 & 11 \\
\hline 2000-2009 & 4 & 13 & 1 & 3 & 5 & 4 & 0 & 1 & 31 \\
\hline \multirow[t]{2}{*}{ 2010-2016 } & 9 & 28 & 3 & 7 & 4 & 1 & 1 & 3 & 55 \\
\hline & 17 & 53 & 5 & 14 & 10 & 6 & 1 & 8 & 114 \\
\hline
\end{tabular}

1. Ces articles présentent pour la grande majorité des résultats qualitatifs et quantitatifs. Seulement deux articles portent uniquement sur la portion qualitative des résultats. Puisque ces deux derniers utilisaient les entretiens dans leur section qualitative, ils ont été considérés dans cette même section pour notre analyse. 
dispositifs afin d'appréhender leurs objets d'étude. Quelques études à devis mixte composent notre corpus, dont le nombre augmente au fil du temps, pour culminer entre 2010 et 2016.

\subsection{Au cœur de la méthodologie qualitative, trois grands dispositifs de collecte de données}

Les prochaines sections visent la description des usages des trois dispositifs de collecte de données principalement utilisés dans les articles de la revue: les entretiens, l'analyse documentaire et l'observation.

\subsubsection{Les entretiens}

La grande majorité (91) des articles consultés ont eu recours aux entretiens, généralement individuels, comme principale source de connaissances. Le plus souvent, ces articles évoquent l'exploration ou la reconstruction de l'expérience, des perceptions ou des trajectoires d'acteurs clés par la conduite d'entretiens avec des personnes délinquantes ou des victimes (directes ou non) d'actes criminels. D'autres ont plutôt utilisé l'entretien afin d'appréhender le fonctionnement de systèmes ou d'institutions ou encore, le fondement de certaines pratiques. Des entretiens avec des policiers, des acteurs clés du système judiciaire (avocats et juges) et différents intervenants œuvrant auprès de clientèles marginalisées ou délinquantes ont alors été menés.

Environ une vingtaine d'auteurs ont précisé les raisons justifiant le recours à l'entretien en rappelant les avantages de la technique en lien avec les objectifs de recherche. Par exemple, l'étude de Frigon (2010) portant sur l'expérience de la danse en prison justifie le recours à la technique d'entretien par ses avantages et par un argument épistémologique :

Nous avons choisi l'entretien [...] car il nous offre une plus grande ouverture, flexibilité et permet l'exploration de sujets complexes. Cette technique part du postulat que l'enquêtée est la mieux placée pour parler du sujet car elle le vit, et l'enquêteur a un rôle de facilitateur. (p. 181)

Les modalités entourant la conduite des entretiens sont peu rapportées dans les articles. Lorsque les entretiens sont individuels, quelques auteurs mentionnent avoir mené des entretiens non directifs, préférant le «climat naturel qui favorise l'expression des sujets» (Baril, 1979, p. 68) et suscitant l'émergence de nouveaux thèmes non imposés par le chercheur (Dozois, Lalonde et Poupart, 1984). 
Lorsqu'il est question d'entretiens semi-structurés, ce qui concerne le tiers des recherches utilisant l'entretien, les thèmes qui y sont abordés sont souvent soulignés dans la section méthodologique, mais très peu fournissent davantage de détails quant au contenu des entrevues. Larticle de Lavergne, Turcotte et Damant (2008), menée auprès d'intervenants de la protection de la jeunesse, portant sur leurs pratiques dans les situations où il y a des problèmes de violence conjugale et de maltraitance envers les enfants, est un exemple représentatif: «L'entrevue de recherche $[. .$.$] effectuée sur le lieu de travail du participant, abordait$ différents éléments de sa pratique» (p. 252). Deux exceptions sont dignes de mention: F.-Dufour (2015) et Wegrzycka (2007) ont précisé les questions de leurs entretiens menés respectivement auprès de personnes ayant reçu une peine d'emprisonnement avec sursis et de joueurs en traitement.

Enfin, la taille de l'échantillon est une information intéressante à préciser étant donné que la publication d'articles qualitatifs ayant des échantillons volumineux semble être une pratique de plus en plus commune dans certaines revues anglophones (Copes, Brown et Tewksbury, 2011). Toutefois, les articles composant notre corpus et citant ces informations ne semblent pas s'inscrire dans cette tendance, bien au contraire. À l'instar de recherches ayant porté sur des questions d'ordre méthodologique (Baribeau et Royer, 2012; Mason, 2010), le plus souvent, les échantillons sont composés de 10 à 30 participants. Et ces échantillons n'ont pas tendance à augmenter avec les années. Par ailleurs, sept études, dont plus de la moitié ont été publiées depuis les années 2000, et où des entretiens semi-dirigés ont été menés, ont eu recours à des échantillons de très grande taille. Ceux-ci regroupent plus d'une centaine de participants. Étonnamment, ces auteurs n'expliquent pas les raisons justifiant la pertinence de recourir à de tels échantillons.

\subsubsection{L'analyse documentaire}

Le corpus à l'étude comprend 34 articles ayant eu recours à une analyse documentaire, comme méthode principale (14) ou complémentaire. Ces articles ont été publiés, pour la plupart, depuis les années 2000. Lorsqu'elle est la technique principale, l'analyse documentaire vise à documenter des phénomènes ou, encore, comme le document s'impose en tant que seul témoin d'activités particulières d'un passé lointain ou rapproché (Cellard, 1997), à reconstruire des phénomènes ou perspectives. C'est le cas de l'étude de Bernier et Cellard (1996) portant sur 
les représentations des femmes ayant perpétré un meurtre à la fin du $\mathrm{XIX}^{\mathrm{e}}$ siècle et au tournant $\mathrm{du} \mathrm{Xx}^{\mathrm{e}}$. L'analyse documentaire a été effectuée à partir de transcriptions de sept procès de femmes reconnues coupables de «maricide» et a permis de questionner le discours des hommes de loi à partir duquel ces femmes ont été jugées.

Dans certains cas, même si les auteurs ne le précisent pas toujours, l'analyse documentaire s'avère un compromis à défaut d'avoir accès à des acteurs clés ou à des données directes. L'étude de Tanner (2012) sur la participation d'un groupe armé à des événements violents en ex-Yougoslavie dans les années 1990 représente un bon exemple. L'analyse documentaire est basée sur des transcriptions judiciaires de procès ainsi que sur un document audiovisuel, seul connu sur le sujet. Par ailleurs, Tanner (2012) justifie son choix de dispositif, mais également son choix pour la recherche qualitative, par la pertinence de son matériau pour l'étude de l'implication de ce groupe et sur la criminalité de masse:

Ce matériel représente une grande valeur pour le chercheur en sciences sociales. Il documente, de l'intérieur, les faits et gestes de cette unité et ouvre une fenêtre originale sur les dynamiques sociales qui la caractérisent. (p. 33)

Les sources documentaires utilisées par les auteurs des études consultées sont variées, dont environ le tiers sont des articles de journaux. Par exemple, l'étude de Sacco (2000), reposant sur une analyse documentaire de 244 articles parus dans les années 1990 dans la presse canadienne, a permis de mettre en exergue le traitement médiatique des statistiques portant sur la criminalité et la victimisation. Des documents issus des instances gouvernementales, dont des commissions d'enquête, des textes de loi, des rapports d'enquêtes policières et des dossiers criminels, figurent également comme sources de données, mais dans une proportion moins grande. Enfin, un peu plus du tiers seulement de ces articles mentionne avec précision le nombre de documents sur lesquels l'analyse a été menée, laissant parfois le lecteur s'interroger sur l'ampleur du corpus.

\subsubsection{L'observation}

Peu d'articles composant notre échantillon ont eu recours à l'observation comme technique principale. Seulement cinq l'ont fait, et ce, depuis les années 1970. Ceci est particulièrement intéressant puisque, tel qu'il 
est mentionné en première partie, la technique de l'observation participante a largement contribué à faire connaître et à populariser l'approche ethnographique aussi bien en sociologie qu'en anthropologie, en plus d'être particulièrement valorisée et utilisée par certains professeurs de l'École au début des années 1970.

L'étude de Jobard (2005), portant sur les interventions policières dans les zones dites de non-droit, représente l'une des quelques études publiées dans la revue reposant sur les observations comme dispositif principal de collecte de données. L'auteur a utilisé ses notes de terrain détaillées, qualifiées de thick description, afin d'exemplifier un phénomène, ici le recours à la violence des policiers.

D'autres auteurs se sont montrés davantage évasifs quant à leurs dispositifs de collecte de données, laissant même parfois deviner au lecteur qu'il s'agit effectivement d'observations. Celles-ci sont alors décrites comme des voyages sur le terrain ou de l'accompagnement d'acteurs privilégiés dans leurs activités.

Les auteurs ayant justifié leur choix pour l'observation participante ont souligné, à l'instar de Desmazières (2016) dans son étude sur la lutte anti-contrefaçon, la proximité qu'elle permet avec l'objet d'étude:

Cette méthode d'observation directe offre l'avantage d'appréhender les pratiques telles qu'elles se produisent, elle est également particulièrement adaptée à la récolte de données non verbales telles que des comportements ou des conduites, et produit un matériau plutôt spontané [...], l'appréhension de la complexité sociale des dispositifs sécuritaires nécessite un contact direct avec les pratiques de ses acteurs. (p. 57)

Cette proximité que permet l'observation est souvent présentée dans les études comme donnant la possibilité d'appréhender des pratiques et l'expérience subjective ainsi que d'étudier en profondeur des interactions sociales. Il est apparu que lorsque les observations complètent les données collectées par un autre dispositif, ce qui est presque toujours le cas dans notre échantillon, les observations permettent de se familiariser avec des milieux tout en créant des contacts et d'étudier des pratiques afin d'être en mesure de saisir le sens de celles-ci dans leur contexte. Ce contenu ainsi amassé peut ensuite soutenir la conduite d'entretiens en profondeur. L'étude de Huot et Giroux (1979) s'intéressant aux mécanismes du système pénal et aux façons dont les citoyens semblent (mal) servis par la justice en constitue un exemple. La façon dont les auteurs décrivent l'importance de l'observation est explicite : 
Pour ce faire, nous avons, pendant plus de cinq mois, à raison de deux à quatre jours par semaine, fréquenté les tribunaux [...]; ce qui nous a permis de nous familiariser avec le monde hermétique de la justice. Nous nous sommes initiés peu à peu au langage spécialisé des experts [...] aux pratiques en vigueur, aux astuces et aux procédures judiciaires, à la prose rigoureuse des articles de loi. Nous avons pu de cette façon saisir la signification et la portée des diverses étapes du procès, le sens des jeux de coulisse et des apartés entre les différents acteurs. (p. 43)

\subsection{Justifications du choix de la méthode qualitative}

Il existe différentes justifications auxquelles ont eu recours les auteurs des articles composant notre corpus afin de fonder le choix d'un devis qualitatif. Ces justifications sont de trois ordres: épistémologique, méthodologique et éthico-politique (Poupart, 1997). La première permet l'analyse des réalités sociales selon la perspective des acteurs sociaux, perspective essentielle où elle donne la possibilité d'éclairer les conduites et de saisir le sens que celles-ci prennent pour les acteurs. L'argument d'ordre méthodologique situe l'utilité de la méthode dans sa capacité à explorer une réalité sociale en profondeur et dans sa capacité à susciter l'émergence de nouvelles dimensions tout en évitant la préstructuration des discours. Le dernier argument, d'ordre éthicopolitique, vise à décrire les dilemmes vécus par les acteurs et à dénoncer les préjugés, iniquités ou toutes autres pratiques discriminatoires.

Les justifications rapportées dans la section méthodologique sont peu nombreuses. Globalement, ces auteurs ont mentionné que la méthode qualitative permet d'avoir accès à la perspective des acteurs ou, encore, de mieux s'approcher de la réalité subjective de ceux-ci (argument épistémologique). Si certains évoquent l'argument méthodologique où le qualitatif permet d'explorer une réalité sociale en profondeur, d'autres choisissent de justifier leur démarche en rappelant la rigueur de cette dernière. Par exemple, dans son article sur le processus de changement des hommes détenus purgeant de longues peines, Casoni (2010) précise:

Bien que préliminaires, les analyses effectuées à ce jour possèdent les caractéristiques essentielles d'une méthodologie qualitative rigoureuse et d'une analyse par trajectoire de vie, c'est-à-dire qu'elles ont été faites selon un mode inductif, sont rigoureuses et ancrées dans les données empiriques recueillies. (p. 358) 
Loin d'être absentes du corpus, les justifications d'ordre éthico-politique touchent aux visées des recherches et sont souvent énoncées dans les conclusions des articles dans lesquelles les auteurs précisent les retombées de leurs recherches. Par exemple, les études sur les victimes menées par Huot et Giroux (1979) et Baril et al. (1983) cherchent à décrire leur expérience de victime et leur cheminement par les tribunaux. Denis (2003) et Bellot, Rivard et Greissler (2010) montrent comment l'expérience de la rue et du squeegee sert d'espace d'intégration pour des jeunes et comment certaines mesures peuvent contribuer à les marginaliser davantage. Ces quelques exemples permettent d'illustrer un souci de pragmatisme chez les auteurs.

Puisque les méthodes qualitatives sont bien établies en criminologie comme en sciences sociales, il semble que les chercheurs ne se sentent plus contraints de les justifier en fonction des insuffisances de la méthode quantitative, telle qu'était bien souvent la pratique dans les années 1970. Au contraire, les justifications retrouvées tendent à expliquer en quoi la méthodologie qualitative permet d'appréhender l'objet d'étude judicieusement.

\subsection{Approches théoriques de la section méthodologique}

À l'image des approches méthodologies recensées par Royer et al. (2009), les justifications théoriques abordées dans les sections méthodologiques $^{6}$ des articles consultés ne sont que peu explicitées. Bien que la grande majorité des auteurs resituent leur objet par rapport à la littérature existante, l'ancrage théorique de la méthodologie n'est abordé que dans une vingtaine articles. Ces perspectives théoriques s'inscrivent alors à titre de justifications pour la méthodologie, mais surtout comme une façon de camper l'angle dans lequel l'objet d'étude sera traité.

Compte tenu de l'influence de la tradition de Chicago sur le plan méthodologique, il n'est pas étonnant que plusieurs de ces auteurs y réfèrent directement. C'est le cas de Jaspart (2016) dont l'article porte sur l'impact de l'enfermement sur le rapport au corps des jeunes:

C'est ainsi que nous avons choisi de pousser la porte de ces institutions [d'enfermement] en empruntant une méthodologie ethnographique ancrée dans une approche interactionniste. (p. 249)

6. À ne pas confondre avec le cadre conceptuel ou les approches théoriques utilisées dans la façon d'aborder l'objet d'étude. 
Des postures, campées en sociologie, comme l'interactionnisme symbolique et l'ethnométhodologie ont également été rapportées. Quelques auteurs ont cité une perspective constructiviste comme Cauchie, Corriveau, Tracqui et Cyr (2015), dans leur article portant sur les enjeux définitionnels des gangs de rue. De plus, dans son article portant sur la violence à laquelle font face les femmes de militaires, Harrison (1997) campe sa position dans un courant marxiste et de la sociologie féministe:

Nous avons utilisé la méthodologie sociologique féministe de l'ethnographie institutionnelle [...]. Cette méthodologie ethnographique institutionnelle a pour fondement la théorie de l'aliénation de Karl Marx, laquelle implique que tous ceux qui ont été exclus de «l'élaboration d'une idéologie, d'un savoir et d'une culture» se retrouvent piégés dans des systèmes qui définissent sans appel leur mode de vie. (p. 29)

Par ailleurs, les perspectives féministes servent également de point d'ancrage à quelques articles revus. Beaucoup moins fréquents, et sans surprise comme les méthodes qualitatives ont davantage été utilisées dans des perspectives marquées par la sociologie et l'anthropologie, deux articles rapportent des positions issues de la psychologie.

Enfin, il est intéressant de noter que ces considérations pour l'approche théorique sont rapportées plus fréquemment dans les articles datant de la dernière décennie. Toutefois, il n'y a guère trace des débats dans ces articles, sans doute parce que ces débats ont décliné à partir des années 1990. Les auteurs continuent néanmoins de faire référence aux diverses perspectives qui ont marqué l'histoire de la criminologie à l'École comme ailleurs, par exemple en rattachant leurs travaux au courant de la réaction sociale, à la perspective constructiviste ou, encore, par l'utilisation de certains concepts liés à ces perspectives comme ceux de réaction sociale (Baril et al., 1983) ou de stigmatisation (Gras, 2003).

\subsection{La question de la généralisation des résultats}

Vingt-huit articles, dont la majorité a été publiée récemment, sont qualifiés d'exploratoires. Peu de détails quant aux raisons appuyant ce choix sont toutefois rapportés. Certains auteurs utilisent le terme «exploratoire» d'une façon près de la sémantique du terme, soit lorsqu'une étude permet de mettre en relief certains aspects de l'objet d'étude mais que davantage de recherches sont nécessaires afin d'arriver à mieux saisir celui-ci. L'extrait suivant, d'une étude menée auprès des jeunes de la rue, l'illustre adéquatement: 
Une approche qualitative exploratoire montre non seulement la nécessité de parfaire nos connaissances dans un champ encore peu étudié mais aussi l'intérêt de mieux comprendre la manière complexe dont les structures liées à l'emploi s'articulent à divers systèmes de sens. (Karabanow, Hugues et Kidd, 2010, p. 11-12)

D'autres auteurs ont appuyé une des limites de leur recherche sur le fait que celle-ci était, avant tout, exploratoire. Le terme exploratoire signifierait alors que le matériel ou les analyses sont rudimentaires et ne permettent pas de jeter des conclusions solides sur l'objet d'étude. Certains auteurs insistent alors sur le fait que puisque leur recherche est exploratoire, les conclusions ne peuvent être généralisées. C'est le cas de la recherche de Collins (2012) portant sur le processus de réinstallation au Canada de jeunes réfugiés séparés de leurs familles. «En raison de l'objectif exploratoire de cette recherche, les résultats ne peuvent être généralisés à tous les enfants réfugiés séparés» (Collins, 2012, p. 56). Plusieurs auteurs des études consultées se montrent ainsi prudents quant à la portée de leurs résultats, mais l'absence d'explication quant à cette limite qu'ils établissent laisse perplexe.

\section{Conclusion}

C'est à partir des années 1970 et 1980 que les méthodes qualitatives se sont développées à l'École de criminologie et sont devenues une formule de recherche légitime et crédible sur le plan scientifique. La revue Criminologie étant étroitement liée à l'École, il est peu étonnant d'y retrouver plusieurs articles présentant des recherches fondées sur ces méthodes.

L'examen de ces articles permet de dresser le portrait des usages de la méthodologie qualitative, d'exemplifier la façon dont sont rapportés ces usages et de cerner certaines tendances. En premier lieu, l'approche prédominante est indéniablement celle des entretiens, celles de l'analyse documentaire et de l'observation étant moins fréquemment utilisées. Dans plusieurs cas, les approches sont combinées ou employées dans le cadre de recherches adoptant un devis mixte qualitatif et quantitatif. En second lieu, bien que la sociologie interactionniste et la sociologie des représentations aient joué un rôle important dans l'implantation de ces méthodes en criminologie, celles-ci ont été et sont pratiquées selon diverses orientations théoriques et pour appréhender une diversité d'objets, certains plus anciens, d'autres plus nouveaux comme les ques- 
tions de sécurité en fonction de l'évolution de l'École et de la discipline. Deux grands angles ressortent dans la manière d'appréhender les objets: celui de comprendre les perspectives et les expériences des acteurs individuels ou collectifs dans les processus d'implication dans la déviance ou lors de leur passage dans le système pénal; celui d'appréhender les modes de régulation sociale par l'analyse notamment des pratiques professionnelles, du fonctionnement des institutions et des transformations plus générales dans le champ sociopénal.

La présentation de la méthodologie utilisée dans les recherches publiées dans la revue est dans certains cas détaillée, dans d'autres, sommaire. Bien que les questions d'ordre méthodologique puissent être abordées ailleurs que dans la présentation de la méthodologie, par exemple lors de l'analyse des données, il demeure qu'une description suffisamment étoffée de la méthodologie mise en œuvre dans le cadre d'une recherche est nécessaire pour apprécier les connaissances produites et leur transférabilité. Cette description participe en outre à la diffusion de savoirs pratiques ou réflexifs issus de démarches concrètes. Il y a lieu de s'interroger sur la place que devrait occuper la méthodologie dans les comptes rendus de recherche et d'encourager la poursuite des réflexions en ce sens, sans pour autant tomber dans un formalisme méthodologique.

Nous avons souligné que le débat concernant les méthodes qualitatives et quantitatives s'est estompé à partir du milieu des années 1980, entre autres parce que ces méthodes sont vues comme traversées par des interrogations théoriques et épistémologiques communes, que l'accent est mis depuis longtemps sur leur complémentarité et que, dans les dernières décennies, il y a eu un déplacement des réflexions dans le champ même des méthodes qualitatives, celles-ci se situant sur leur propre terrain. Sans doute cela explique-t-il en partie que les présentations de la méthodologie qualitative dans la revue Criminologie ne s'articulent plus autour du débat qualitatif et quantitatif.

Cependant, dans la littérature anglo-saxonne en provenance des États-Unis et de l'Angleterre, plusieurs chercheurs en qualitatif soulignent qu'il y aurait résurgence de ce débat. Cette résurgence se traduirait par un retour à une forme de positivisme «dur», laissant peu ou moins de place aux méthodes qualitatives, et aurait pour conséquences des difficultés sur le plan de l'obtention de subventions de recherche et de la publication des travaux. Ainsi, Copes et al. (2011) rapportent qu'entre les années 2000 à 2009, seulement 4\% des études publiées 
dans les revues criminologiques américaines considérées comme les plus prestigieuses seraient de type qualitatif. Selon Travers (2013), ce faible pourcentage doit être interprété avec prudence, les raisons du choix des articles par ces revues ayant été peu étudiées, et pourrait conduire à sous-estimer la place des méthodes qualitatives au sein de la criminologie des pays considérés. Nous n'avons pas observé une telle tendance dans la revue Criminologie, le nombre d'articles usant des méthodes qualitatives ayant au contraire augmenté.

Après un siècle d'existence en sociologie et un demi-siècle à l'École de criminologie, en criminologie et dans la revue Criminologie, la pertinence de ces méthodes n'est plus à démontrer. Il incombe aux chercheurs recourant aux méthodes qualitatives de mener à bien leur recherche selon leurs spécificités. Il ne manque pas d'outils pour y parvenir. Les exemples de recherches qualitatives en criminologie sont nombreux et il y a une littérature abondante autant sur les fondements de ces méthodes que sur la manière de les pratiquer, y compris en criminologie puisque ces méthodes sont devenues un champ d'étude en soi comme dans d'autres disciplines des sciences sociales. Bien qu'il n'y ait pas d'articles portant spécifiquement sur la méthodologie qualitative dans la revue Criminologie, le présent numéro de la revue constitue un premier effort dans cette direction.

\section{Références}

Acosta, F. (1986). Les affaires de ville d'Anjou ou la mise en forme pénale de la corruption politique (Thèse de doctorat inédite). Université de Montréal.

Baribeau, C. et Royer C. (2012). L'entretien individuel en recherche qualitative: usages et modes de présentation. Revues des sciences de l'éducation, 38(1), 23-45.

Baril, M. (1979). Violence et répression: mythe ou réalité? Criminologie, 12(1), 66-77.

Baril, M., Cousineau, M.-M. et Gravel, S. (1983). Quand les femmes sont victimes..., quand les hommes appliquent la loi. Criminologie, 16(2), 89-100.

Bellot, C., Rivard, J. et Greissler, É. (2010). L'intervention par les pairs: un outil pour soutenir la sortie de rue. Criminologie, 43(1), 171-198.

Bernier, J. et Cellard, A. (1996). Le syndrome de la femme fatale: «matricide» et représentation féminine au Québec, 1898-1940. Criminologie, 29(2), 29-48.

Bertrand, M.-A. (1979). La femme et le crime. Montréal, Québec: L’Aurore.

Bertrand, M.-A., Brousseau, G., Gagnon, A., Garigue, V., Gauthier, M., Lallier, M.,... Patenaude, S. (1972). Présentation thématique et objectifs de la recherche. Montréal, Québec: Université de Montréal. 
Blankevoort, V., Landreville, P. et Pires, A. P. (1981). Système pénal et trajectoire sociale. Déviance et Société, 5(4), 319-345.

Brillon, Y. (1980). Ethnocriminologie de l'Afrique noire. Montréal, Québec: Presses de l'Université de Montréal.

Brodeur, J.-P. (1984). La police: mythes et réalités. Criminologie, 17(1), 9-41.

Carrière, P. (1976). Le processus décisionnel de la Commission des libérations conditionnelles (Mémoire de maîtrise inédit). Université de Montréal.

Casoni, D. (2010). L'étude du processus de changement chez des hommes condamnés à de longues peines. Criminologie, 43(2), 351-372.

Cauchie, J.-F., Corriveau, P., Tracqui, H. et Cyr, K. (2015). Des p'tits culs aux gangs de rue. Action concertée et enjeux définitionnels1. Criminologie, 48(2), 65-82.

Cefaï, D. (2003). L'enquête de terrain en sciences sociales. Paris, France: La Découverte.

Cellard, A. (1997). L'analyse documentaire. Dans J. Poupart, J.-P. Deslauriers, L.-H. Groulx, A. Laperrière, R. Mayer et A. P. Pires (dir.), La recherche qualitative: enjeux épistémologiques et méthodologiques (p. 251-271). Boucherville, Québec: Gaétan Morin éditeur.

Chapoulie, J.-M. (1984). Everett C. Hughes et le développement du travail de terrain en France. Revue française de sociologie, 25(4), 582-608.

Charette, Y., Tremblay, P. et Deslauriers-Varin, N. (2018). La trajectoire de la recherche empirique quantitative dans la revue Criminologie (1968-2016): une perspective éditoriale. Criminologie, 51(1), 169-200.

Clough, P. (1992). The end(s) of ethnography: from realism to social criticism. Newbury Park, CA: Sage Publications.

Collins, R. (2012). Repartir à zéro loin de sa terre natale: la réinstallation des Lost Boys and Girls of Sudan au Canada. Criminologie, 45(1), 51-69.

Copes, H., Brown, A. et Tewksbury, R. (2011). A content analysis of ethnographic research published in top criminology and criminal justice journals from 2000 to 2009. Journal of Criminal Justice Education, 22(3), 341-359.

Denis, V. (2003). Pour comprendre la pratique du «squeegee» à Montréal. Criminologie, 36(2), 89-104.

Denzin, N. K. et Lincoln, Y. S. (1994). The handbook of qualitative research. Thousand Oaks, CA: Sage Publications.

Denzin, N. K. et Lincoln, Y. S. (2018). The SAGE handbook of qualitative research (5 éd.). Thousand Oaks, CA: Sage Publications.

Desmazières, A. (2016). Intermédiaire d'intermédiaires: la place du privé dans la lutte anticontrefaçon. Criminologie, 49(1), 51-77.

Dozois, J., Lalonde, M. et Poupart, J. (1984). Dangerosité et pratique criminologique en milieu adulte. Criminologie, 17(2), 25-51.

Ellenberger, H. F. (1965). Criminologie du passé et du présent, leçon inaugurale. Montréal, Québec: Presses de l'Université de Montréal.

Erickson, F. (2018). A history of qualitative inquiry in social and educational research. Dans N. K. Denzin et Y. S. Lincoln (dir.), The SAGE bandbook of qualitative research (5 éd., p. 27-65). Thousand Oaks, CA: Sage Publications. 
F.-Dufour, I. (2015). Le désistement assisté? Les interventions des agents de probation telles que perçues par des sursitaires qui se sont désistés du crimel. Criminologie, 48(2), 265-288.

Finkler, H. W. (1975). Inuit and the administration of criminal justice in the Northwest Territories: The case of Frobisher Bay (Mémoire de maîtrise inédit). Université de Montréal.

Finkler, H. W. (1980). Les inuits et l'administration de la justice: le cas de Frobisher Bay. Montréal, Québec: Les Éditions Hurtubise.

Frigon, S. (2010). La danse en prison, une échappée belle hors des murs? Perspective des artistes et des détenues. Criminologie, 43(2), 179-197.

Gagné, D. (1970). Caractère social et déviance chez les adolescents de milieu ouvrier et aisé (Thèse de doctorat inédite). Université de Montréal.

Garland, D. (1981). Law and ideology in Marxist theory. Sociology, 15(1), 127130.

Glaser, B. G. et Strauss, A. L. (1967). The discovery of grounded theory: Strategies for qualitative research. Chicago, IL: Aldine.

Gras, L. (2003). Show sportif et buffet froid. Interactions sportives et sociales entre détenus et joueurs extérieurs. Criminologie, 36(2), 105-125.

Groulx, L.-H. (1998). Sens et usage de la recherche qualitative en travail social. Dans J. Poupart (dir.), La recherche qualitative: diversité des champs et des pratiques au Québec (p. 1-50). Boucherville, Québec: Gaëtan Morin éditeur.

Harrison, D. (1997). La violence dans la communauté militaire. Criminologie, $30(2), 27-45$.

Huot, L. et Giroux, J. (1979). Le citoyen et la justice. Criminologie, 12(1), 43-56.

Jaspart, A. (2016). Quand les corps adolescents parlent de l'enfermement. Criminologie, 49(1), 247-262.

Jobard, F. (2005). Le nouveau mandat policier: faire la police dans les zones dites de «non-droit». Criminologie, 38(2), 103-121.

Kaminski, D. et Kokoreff M. (2004). Sociologie pénale; système et expérience. Toulouse, France: Érès.

Karabanow, J., Hugues, J. et Kidd, S. (2010). Travailler pour survivre: exploration du travail des jeunes de la rue. Criminologie, 14(1), 7-29.

Laberge, D. (1983). La décroissance récupérée: une nouvelle modalité d'expansion de la gestion pénale. Dans L. Pilon et A. Hubert (dir.), Les enjeux sociaux de la décroissance. Montréal, Québec: Saint-Martin.

Laflamme-Cusson, S. et Baril, M. (1975). La détention des mineurs dans la région de Montréal. Montréal, Québec: Ministère des Affaires sociales.

Lavergne, C., Turcotte, D. et Damant, D. (2008). Cooccurrence de violence conjugale et de maltraitance envers les enfants: points de vue des intervenants de la protection de la jeunesse. Criminologie, 47(2), 247-267.

Le Blanc, M. (1972). La délinquance en milieu aisé: un groupe culture d'adeptes de la drogue. Acta Criminologica, 5(1), 107-181.

Louis-Guérin, C. (1979). Réflexions méthodologiques pour une recherche sur l'opinion publique et l'action sociale: essai de synthèse. Criminologie, 12(1), 24-42. 
Lumsden, K. et Winter, A. (2014). Reflexivity in criminological research: Experiences with the powerful and the powerless. Basingstoke, Angleterre: Palgrave Macmillan UK.

Manseau, H. et Grenier, H. (1979). Les petits commerçants victimes de vol à main armée en quête de justice. Criminologie, 12(1), 57-65.

Mason, M. (2010). Sample size and saturation in $\mathrm{PhD}$ studies using qualitative interviews. Forum: Qualitative Social Research, 11(3). Repéré à http://www. qualitative-research.net/index.php/fqs/article/view/1428/3027

Miles, M. B. et Huberman, A. M. (1994). Analyse des données qualitatives: recueils de nouvelles méthodes. Bruxelles, Belgique: De Boeck-Wesmael.

Nicolas, M. (1974). La suspension de la libération conditionnelle au Canada: une étude exploratoire qualitative (Mémoire de maîtrise inédit). Université de Montréal.

Normandeau, A. et Szabo, D. (1970). Synthèse des travaux: Ier Symposium international de recherche en criminologie comparée, Mont-Gabriel, 30 avril-3 mai 1969. Acta Criminologica, 3(1), 143-170.

Olivier de Sardan, J.-P. (2008). La rigueur du qualitatif. Louvain-la-Neuve, Belgique: Bruylant-Academia.

Pierret, J. (2004). Place et usage de l'entretien en profondeur en sociologie. Dans D. Kaminski et M. Kokoreff (dir.), Sociologie pénale: système et expérience (p. 199-213). Toulouse, France: Érès.

Pires, A. (1993). La criminologie et ses objets paradoxaux: réflexions épistémologiques sur un nouveau paradigme. Déviance et société, 17(2), 129-161.

Pires, A. (1995). À propos des objets en criminologie: quelques réponses. Déviance et société, 19(3), 291-303.

Pires, A (1998). La criminologie d'hier à d'aujourd'hui. Dans C. Debuyst, F. Digneffe, J.-M. Labadie et A. Pires (dir.), Histoire des savoirs sur le crime et la peine: des savoirs diffus à la notion de criminel-né(vol. 1, p. 13-67). Montréal, Québec: Presses de 1'Université de Montréal.

Pires, A. (2004). La recherche qualitative et le système pénal. Peut-on interroger les systèmes sociaux. Dans D. Kaminski et M. Kokoreff (dir.), Sociologie pénale: système et expérience (p. 173-198). Toulouse, France: Érès.

Poupart, J. (1993). Discours et débats autour de la scientificité des entretiens de recherche. Sociologie et sociétés, 25(2), 93-110.

Poupart, J. (1997). L'entretien de type qualitatif: considérations épistémologiques, théoriques et méthodologiques. Dans J. Poupart, J.-P. Deslauriers, L.-H. Groulx, A. Laperrière, R. Mayer et A. P. Pires (dir.), La recherche qualitative: Enjeux épistémologiques et méthodologiques (p. 173-209). Boucherville, Québec: Gaétan Morin éditeur.

Poupart, J. (2004). L'institutionnalisation de la criminologie au Québec: une lecture sociohistorique, Criminologie, 37(1), 71-105.

Poupart, J. (2008). Sociologie de la déviance. L’héritage interactionniste et son actualité. Dans J. Lafontant, et S. Laflamme (dir.), Initiation thématique à la sociologie (p. 237-262). Ottawa, Ontario: Éditions Prise de parole.

Poupart, J. (2011). Tradition de Chicago et interactionnisme: des méthodes qualitatives à la sociologie de la déviance. Recherches qualitatives, 30(1), 178199. 
Poupart, J., Dozois, J. et Lalonde, M. (1982). Dangerosité et pratique criminologique en milieu adulte. Criminologie, 17(2), 25-51.

Poupart, J., et Lalonde, M. (1997). La méthodologie qualitative et la criminologie au Québec, de 1960-1985. Dans J. Poupart, J.-P. Deslauriers, L.-H. Groulx, A. Laperrière, R. Mayer et A. P. Pires (dir.), La recherche qualitative: enjeux épistémologiques et méthodologiques (p. 51-91). Boucherville, Québec: Gaétan Morin éditeur.

Robert, P. et Faugeron, C. (1978). La justice et son public, les représentations sociales du système pénal. Paris, France: Masson.

Royer, C., Baribeau, C. et Duchesne, A. (2009). Les entretiens individuels dans la recherche en sciences sociales au Québec: où en sommes-nous? Un panorama des usages. Recherches qualitatives, Hors Série (7), 64-79.

Sacco, V. (2000). News that counts: Newspaper images of crime and victimization statistics. Criminologie, 33(1), 203-223.

Szabo, D. (1963). Urbanisation et criminalité. Revue de l'Institut de sociologie, 1, $37-52$.

Szabo, D. (1972). Éditorial. Acta Criminologica, 5(1), 3-9.

Tanner, S. (2012). De la sécurité privée à l'armée de destruction massive. La «bande armée» et la criminalité de masse. Criminologie, 45(1), 29-49.

Tardif, G. (1974). Police et politique au Québec. Montréal, Québec: L’Aurore.

Théorêt, B. (1987). L’histoire du contrôle social et pénal des filles au Canada anglais de 1800 à 1930 (Mémoire de maîtrise inédit). Université de Montréal.

Travers, M. (2013). Contemporary comment. The uneasy relationship between criminology and qualitative research. Current Issues in Criminal Justice, 25(3), 551-557.

Wegrzycka, B. (2007). Le prix d'une passion : la carrière du joueur compulsif. Criminologie, 40(1), 31-58.

Wincup, E. (2017). Criminological research: Understanding qualitative methods ( $2^{\mathrm{e}}$ éd.). Londres, Angleterre: Sage Publications.

\section{Qualitative methods in the criminological field: perspectives and use in Criminologie}

ABSTACT - This article describes the status, place, and use of qualitative methods in articles in Criminologie from two complementary perspectives. First, the development of qualitative methods in the School of Criminology of the University of Montréal and in the field of criminology in general is explored. Then we look at the research practices of those using qualitative methods, as exemplified in 114 articles in Criminologie published between 1968 and 2016. Our analysis made it possible to identify the qualitative methods used, to provide examples of how they were described, and to identify trends, some of which are similar to those found in the School of Criminology as well as more generally in the field of criminology.

KEYWORDS - Qualitative methods and Criminologie journal, School of criminology, institutionalization of qualitative methods and criminology, epistemological debates, qualitative approach. 


\section{Los métodos cualitativos en "campo criminológico": Puesta en perspectiva y lugar de estos métodos en la revista Criminologie}

RESUMEN - El objetivo de este artículo es describir el estatus, el lugar y el uso de los métodos cualitativos tal y como aparecen en las investigaciones publicadas bajo el formato de artículo en la revista Criminologie, desde su creación. Dos perspectivas complementarias fueron utilizadas. Primero, el desarrollo de los métodos cualitativos en la Escuela de Criminología de la Universidad de Montreal, y especificamente en criminología, será explorado. Luego, las prácticas de investigación cualitativas difundidas en la revista serán examinadas. Nuestra muestra comprende 114 artículos cualitativos, publicados entre 1968 y 2016. El análisis nos permite realizar un esbozo de los usos de la metodología cualitativa, de ejemplificar la forma en la que son reportados estos usos, y de discernir las tendencias de las cuales algunas son comunes a aquellas observadas en la Escuela de Criminología, pero igualmente, en criminología.

PALABRAS CLAVE - Métodos cualitativos y revista Criminologie, institucionalización de los métodos cualitativos en criminología, debates epistemológicos, aproximaciones cualitativas. 